\section{Efeitos da idade-período e coorte na mortalidade por câncer do ovário no Brasil e suas grandes regiões}

\section{Effects of age-period and cohort on mortality due to ovarian cancer in Brazil and its regions}

\author{
Efectos de la edad-período y cohorte en la \\ mortalidad por cáncer de ovario en Brasil \\ y sus macrorregiones
}

Karina Cardoso Meira 1

Juliano dos Santos 2

Cosme Marcelo Furtado Passos da Silva 3

Aline Alves Ferreira 4

Raphael Mendonça Guimarães 5

Taynãna César Simões 6

doi: $10.1590 / 0102-311 \times 00087018$

\section{Correspondência}

K. C. Meira

Escola de Saúde, Universidade Federal do Rio Grande do Norte. Av. Senador Salgado Filho 2990, Natal, RN 59075-000, Brasil. ninameira87@gmail.com

temporal da mortalidade por câncer do ovário no Brasil e suas grandes regiões, entre o período de 1980 a 2014. Estudo ecológico de tendência temporal em que foram utilizados modelos APC com uma abordagem bayesiana e o método determinístico INLA (Integrated Nested Laplace Approximations) na inferência dos parâmetros. Os dados de mortalidade e os dados populacionais foram obtidos junto ao Departamento de Informática do Sistema Único de Saúde. As taxas de mortalidade por câncer do ovário, segundo região geográfica, foram padronizadas pelo método direito, após correção dos óbitos para causas maldefinidas e diagnóstico incompleto de câncer. No período de estudo, o Brasil apresentou 4,91 óbitos por câncer do ovário por 100 mil mulheres, as regiões Sul $(5,66)$ e Sudeste $(5,70)$ apresentaram as maiores taxas por 100 mil mulheres, e a Região Norte a menor (3,13/100 mil mulheres). Houve aumento progressivo da mortalidade com o avançar da idade em todas as regiões. O modelo APC multivariado de melhor ajuste evidenciou risco positivo de morte no Centro-oeste e Nordeste entre 2010-2014 e, a partir do periodo de 1995-1999, na Região Sul. Observou-se, ainda, risco positivo e significativo de morte para as coortes mais antigas no Sul e Sudeste, e risco reduzido para as coortes mais jovens. O inverso foi observado nas regiões Norte e Nordeste. Evidenciou-se um padrão heterogêneo na evolução temporal da mortalidade por câncer do ovário nas regiões geográficas brasileiras, o que pode estar relacionado aos distintos processos de transição demográfica e epidemiológica vivenciados por estas regiões.

Neoplasias Ovarianas; Mortalidade; Estudos de Séries Temporais
1 Escola de Saúde, Universidade Federal do Rio Grande do Norte, Natal, Brasil.

2 Instituto Nacional de Câncer José Alencar Gomes da Silva, Rio de Janeiro, Brasil.

${ }^{3}$ Escola Nacional de Saúde Pública Sergio Arouca, Fundação Oswaldo Cruz, Rio de Janeiro, Brasil.

4 Instituto de Nutrição Josué de Castro, Universidade Federal do Rio de Janeiro, Rio de Janeiro, Brasil.

5 Escola Politécnica de Saúde Joaquim Venâncio, Fundação Oswaldo Cruz, Rio de Janeiro, Brasil. 6 Instituto René Rachou, Fundação Oswaldo Cruz, Belo Horizonte, Brasil. 


\section{Introdução}

O câncer do ovário representa o sétimo câncer mais incidente e a oitava causa de morte por câncer em mulheres no mundo. Em 2012, segundo o GLOBOCAN 1, estimaram-se 239 mil casos novos (6,1 casos novos/100 mil mulheres) e 152 mil óbitos (3,8 óbitos/100 mil mulheres). Para o mesmo ano, ainda segundo o GLOBOCAN, o Brasil registrou incidência de 5,6 casos novos/100 mil mulheres e 2,7 óbitos/100 mil mulheres. O aumento da incidência esteve associado com as mudanças na estrutura etária da população, seu comportamento reprodutivo e hábitos e estilo de vida 1,2. Ainda, podem sofrer o efeito do período das mudanças da classificação histológica da doença, assim como, da ampliação do acesso aos exames diagnósticos, levando ao sobrediagnóstico 3,4. A respeito da mortalidade, além do diagnóstico tardio 5 , considera-se os mesmos fatores de risco da incidência, uma vez que a letalidade por este câncer é alta e que as inovações diagnósticas e terapêuticas não conseguiram promover aumento significativo na sobrevida, pois grande proporção desta doença é diagnosticada em estágio avançado devido ao seu caráter insidioso e a inexistência de um exame diagnóstico que permita seu uso para o rastreamento em nível populacional 4,5.

O aumento da exposição aos fatores de risco citados antes, concomitante à redução da exposição aos agentes infecciosos associados a certos cânceres devido às melhorias nas condições socioeconômicas e acesso aos serviços de saúde, trouxe à tona um fenômeno classificado como transição do câncer, correlato à transição epidemiológica, conforme documentado por Bray et al. 6, e descrito como tendo relação direta com o Índice de Desenvolvimento Humano (IDH). E assim, nos países com baixo IDH observou-se maiores taxas de incidência e mortalidade por cânceres associados às infecções, enquanto que nos países com alto IDH há redução destes e aumento de cânceres associados às mudanças nos hábitos e estilo de vida, inclusive aos relacionados ao comportamento reprodutivo. Por conta disso, há disparidades na incidência e mortalidade por câncer do ovário entre os países desenvolvidos e em desenvolvimento, sendo que as maiores taxas de incidência e mortalidade ocorrem nos países nórdicos, Estados Unidos e Canadá 1. Esses países completaram sua transição demográfica apresentando baixa taxa de fecundidade, acompanhada de mudanças significativas no comportamento reprodutivo e hábitos alimentares. Situação oposta é verificada na África e sudoeste da Ásia, regiões com menores taxas de incidência e mortalidade por essa doença 1.

No Brasil, para 2018, foram estimados 6.150 casos novos de câncer do ovário para todo o país (5,79 casos/100 mil mulheres) ${ }^{7}$. Por se tratar de um câncer relativamente raro, o seu padrão de evolução é pouco estudado no país. No entanto, o trabalho de Barbosa 8 identificou aumento das taxas de mortalidade por câncer do ovário no período de 1996 a 2010.

Os principais fatores associados ao câncer do ovário podem ser classificados como história familiar (alterações nos genes Brca1 e Brca2), fatores reprodutivos (nuliparidade, lactação, uso de anticoncepcional oral, ligadura de trombas e ooforectomia), fatores relacionados aos hábitos e estilo de vida (tabagismo, aumento do consumo de carnes e gorduras, inatividade física) e exposição ocupacional (asbestos) 9,10,11. A importância dos fatores de risco e proteção no processo de carcinogênese do câncer do ovário depende do seu tipo histológico. Os três tipos histológicos mais incidentes são o câncer epitelial do ovário, câncer de células germinativas e os tumores de células do cordão estromal sexual 12 . Dentre esses, destaca-se o epitelial, responsável por 90\% dos casos novos, ampliando sua incidência a partir dos 40 anos de idade 10,11.

Os fatores reprodutivos apresentam um papel de destaque na história natural deste tipo de câncer. Um estudo realizado na Coreia do Sul em 201011 apontou que a fração atribuível populacional (FAP) associada à nuliparidade, nunca ter amamentado, não ter realizado ligadura de trompas e nunca ter usado anticoncepcional oral foi de $82 \%$. Do mesmo modo, no Reino Unido, uma pesquisa avaliando a mortalidade e a incidência por câncer do ovário identificou maiores taxas em mulheres nascidas entre 1900 e 1920, e redução progressiva nestes indicadores em mulheres nascidas após a década de 194012. O mesmo perfil foi verificado em estudos realizados em países da Europa, Canadá, China e Estados Unidos 12,13,14. Acredita-se que esse cenário seja devido às mudanças no comportamento reprodutivo das mulheres, redução da taxa de fecundidade, uso de anticoncepcional oral e realização da cirurgia de ligadura de trompas 12,13,14.

Esses estudos foram realizados avaliando o efeito da idade, período e coorte (APC) na evolução temporal das taxas de incidência e mortalidade do câncer do ovário. Esta análise tem como vantagem 
permitir levantar hipóteses sobre a evolução temporal da mortalidade e incidência de doenças e agravos à saúde, possivelmente relacionada a alterações no nível de exposição populacional a fatores de risco ou proteção (efeitos de coorte) ou a mudanças nos métodos diagnósticos, tratamentos propostos, acesso aos serviços de saúde e melhoria na certificação dos óbitos (efeitos de período) 15,16.

Por todas essas evidências, acredita-se que possa haver, para a tendência da mortalidade por câncer de ovário no Brasil, disparidades no efeito de período e coorte de nascimento entre as grandes regiões geográficas. Visto que as mudanças geracionais nos hábitos ligados aos fatores reprodutivos, assim como, o envelhecimento populacional e o acesso às políticas de saúde sexual e reprodutiva ocorreram de maneira diferenciada nessas regiões e com certa descontinuidade no tempo, sugerindo a necessidade de modelos que se adaptem a esta condição, de forma a obter uma boa capacidade preditiva. Nesse contexto, este estudo tem por objetivo analisar a evolução temporal da mortalidade por câncer do ovário no Brasil e suas grandes regiões, segundos os efeitos APC, entre 1980 e 2014.

\section{Materiais e métodos}

\section{Desenho do estudo e fonte de dados}

Estudo ecológico de tendência temporal no qual se avaliou os óbitos por câncer do ovário no Brasil e suas regiões geográficas, no período de 1980 a 2014. Os dados de mortalidade foram obtidos junto ao Sistema de Informações sobre Mortalidade (SIM), do Ministério da Saúde. No período analisado, estiveram em vigor a nona (CID-9) e a décima (CID-10) revisões da Classificação Estatística Internacional das Doenças e Problemas Relacionados à Saúde, sendo utilizadas as codificações 183 (CID-9) e C56 (CID 56.0-56.9) (CID-10). A população em estudo foi constituída por mulheres com idades de 20 ou mais anos. Os dados populacionais foram obtidos junto ao Instituto Brasileiro de Geografia e Estatística (IBGE), com base nos censos populacionais de 1980, 1991, 2000 e 2010. Para os anos intercensitários, foram usadas estimativas, igualmente disponibilizadas pelo IBGE. E assim, por tratar-se de dados de acesso livre no qual não há identificação dos sujeitos, o presente trabalho não foi submetido ao Comitê de Ética em Pesquisa.

Os registros de óbitos por câncer do ovário foram corrigidos em duas etapas: (1) redistribuição proporcional por ano e faixa etária dos óbitos classificados como causas maldefinidas, utilizando-se a metodologia proposta pela Organização Mundial da Saúde (OMS) 17: e (2) redistribuição proporcional por ano e faixa etária dos registros classificados como diagnóstico incompleto de câncer geral: 195 (CID-9) e C76 (CID-10); 196 (CID-9) e C77 (CID-10); 197 (CID-9) e C78 (CID-10); 198 (CID-9) e C79 (CID-10); 199 (CID-9) e C80 (CID-10) 18.

As taxas de mortalidade por câncer do ovário foram calculadas, faixa etária e região geográfica por 100 mil mulheres, por grupos etários de cinco anos. As taxas truncadas para idades em intervalo aberto (80 anos e mais) foram calculadas por ano. Obtidas as taxas por faixa etária e idades do intervalo aberto, padronizou-se as taxas quinquenais pelo método direito, tendo como população padrão a proposta por Segi e modificada por Doll \& Hill 19.

Nas análises do efeito APC optou-se por trabalhar com faixas etárias e períodos de observação agrupados em intervalos de cinco anos. Foram avaliadas as faixas etárias a partir dos 20-24 anos, até 80 anos e mais, devido ao excesso de zeros nas faixas menores, resultando, assim, em $I=13$ faixas etárias, $J=7$ períodos de tempo e $K=I+J-1=18$ coortes de nascimento, variando de 1900 a 1990 . Destaca-se que as faixas etárias ignoradas foram redistribuídas proporcionalmente por ano e faixa etária.

\section{Procedimento de modelagem}

Inicialmente, foi ajustado um modelo idade-período-coorte de nascimento bayesiano univariado (BAPC), a fim de estimar os efeitos temporais globais sobre o risco de óbito por câncer do ovário no Brasil 20. Posteriormente, foram ajustados os modelos BAPC multivariados 21 , em que $y_{i j g}$ e $n_{i j g}$ são o número de óbitos e o número de pessoas em risco no grupo etário $i=1, \ldots, 13$, período $j=1, \ldots, 7$, e por região $g$, respectivamente. Sendo os efeitos de período constantes, tem-se como exemplo o modelo (1): 


$$
\begin{gathered}
y_{i j g} \sim \text { Poisson }\left(n_{i j g} \exp \left(\varepsilon_{i j g}\right)\right) \\
\varepsilon_{i j g}=\mu_{g}+\alpha_{i g}+\beta_{j}+\gamma_{k g}+z_{i j}
\end{gathered}
$$

sendo $\mu_{g}$ a média global específica por região $g, \alpha_{i g}$ o efeito do grupo etário $i$ para a região $g, \beta_{j}$ o efeito de período $j, \gamma_{k g}$ o efeito da coorte de nascimento $k$ para a região $g$, e $z_{i j} \sim N\left(0, \delta^{-1}\right)$ os efeitos aleatórios para ajustar para superdispersão (variabilidade extra) 20. Os demais modelos ajustados neste trabalho são variações do modelo (1) e estão listados no Quadro 1.

Para assegurar identificabilidade do intercepto $\mu$, tem-se a restrição:

$$
\sum_{i=1}^{I} \alpha_{i}=j=1 j \beta j=k=1 K \gamma k=0
$$

para cada categoria de região. No entanto, devido ao relacionamento linear entre as variáveis temporais, $K=I-i+j$, os efeitos de idade, período e coorte são ainda não identificáveis.

Sob a abordagem bayesiana, prioris de suavização gaussianas independentes foram usadas para os efeitos temporais principais (primeiras diferenças) e uma priori uniforme foi atribuída a $\mu$. A não identificabilidade dos parâmetros latentes permanece, mas não requer restrições adicionais como nos modelos clássicos devido às prioris escolhidas. Os efeitos temporais têm como prioris passeios aleatórios de primeira (RW1), que penalizam desvios de um modelo em que todos os parâmetros são constantes $\left(\alpha_{1}=\ldots=\alpha_{I}, i=2, \ldots, I\right)^{21}$ :

$$
R W 1: \alpha_{i} \sim N\left(\alpha_{i-1}, \kappa^{-1}\right),(2)
$$

sendo $\kappa$, o parâmetro de suavização (precisão). Neste estudo foram atribuídas hiperprioris não informativas aos parâmetros de precisão $(G(1,0.00005))$ e prioris uniformes independentes a $\alpha_{1}$ e $\alpha_{2}$.

Para a inferência dos parâmetros, foi utilizado o método determinístico INLA (Integrated Nested Laplace Approximations), uma alternativa ao MCMC (Monte Carlo via Cadeias de Markov) em modelos gaussianos latentes 22. O Critério de Informação Deviance (DIC) e o escore logarítmico (log-score) foram usados na comparação dos modelos. Ambos são negativamente orientados, no sentido de que quanto menores são os valores, melhor é o ajuste do modelo 23 . As análises foram feitas no software estatístico R (http://www.r-project.org), com os pacotes inla e bapc.

\section{Quadro 1}

Proposta de modelos multivariados considerando os efeitos temporais, superdispersão, efeito aleatório e heterogeneidade por região geográfica.

\begin{tabular}{|l|c|}
\hline Modelo APC & Preditor linear \\
\hline Modelo 1 & Efeitos conjuntos para as cinco regiões \\
\hline Modelo 2 & Efeitos de idade específicos por regiões \\
\hline Modelo 3 & Efeitos de período específicos por regiões \\
\hline Modelo 4 & Efeitos de coorte específicos por regiões \\
\hline Modelo 5 & Efeitos de idade e período específicos por regiões \\
\hline Modelo 6 & Efeitos de idade e coorte específicos por regiões \\
\hline Modelo 7 & Efeitos de período e coorte específicos por regiões \\
\hline
\end{tabular}




\section{Resultados}

No período estudado foram registrados 64.473 óbitos por câncer do ovário no Brasil, representando uma taxa média ajustada de 4,24 óbitos por 100 mil mulheres. Após o processo de correção, houve um aumento de $15,74 \%$ (74.619) nos óbitos, correspondendo a uma taxa de mortalidade média ajustada de 4,91 óbitos por 100 mil mulheres. As taxas mínima e máxima foram, respectivamente, 3,61 e 5,62 óbitos por 100 mil mulheres. As maiores taxas de mortalidade médias por 100 mil mulheres ocorreram nas regiões Sul (5,66 óbitos) e Sudeste (5,70 óbitos), e a menor taxa ocorreu na Região Norte (3,13 óbitos/100 mil mulheres). Esse perfil foi mantido em todos os quinquênios estudados (Figura 1).

As taxas de mortalidade no Brasil e em suas regiões evidenciaram tendência ascendente com o avançar da idade, sendo as maiores taxas na faixa etária de 80 e mais anos (Figura 1a). Segundo os períodos calendário, observou-se aumento nas taxas médias padronizadas, sendo que as menores ocorreram no início da série histórica (1980-1984) e as maiores no final (2010-2014). No Brasil como um todo, e em todas as regiões geográficas, com exceção das regiões Sul e Sudeste, nas quais as maiores taxas foram observadas, respectivamente, nos períodos de 1995-1999 e 1990-1994, com

\section{Figura 1}

Taxa de mortalidade média para câncer de ovário, segundo efeitos temporais (idade, período e coorte de nascimento), para as regiões do Brasil, no período de 1980 a 2014.

1a) Idade

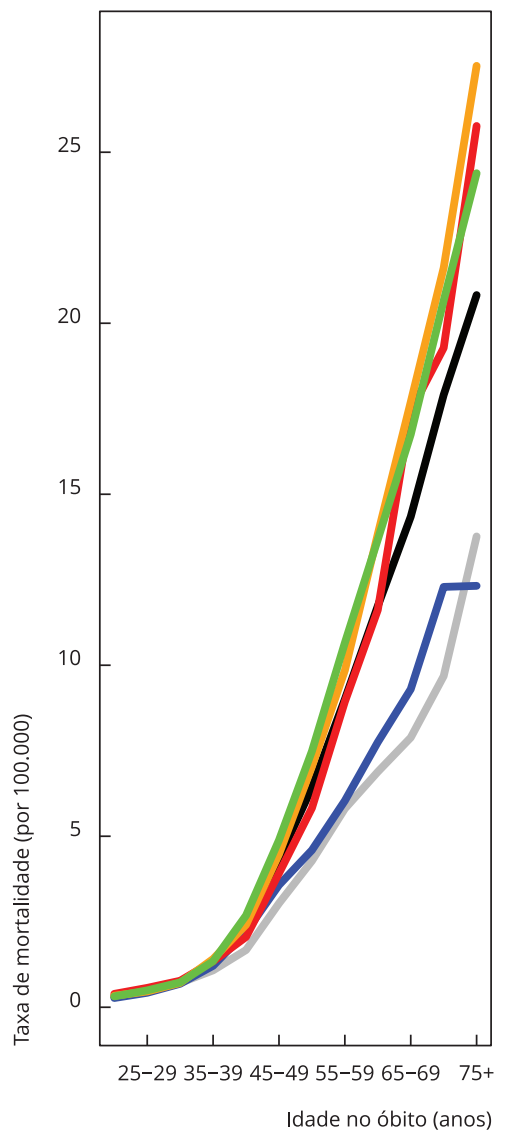

1b) Período

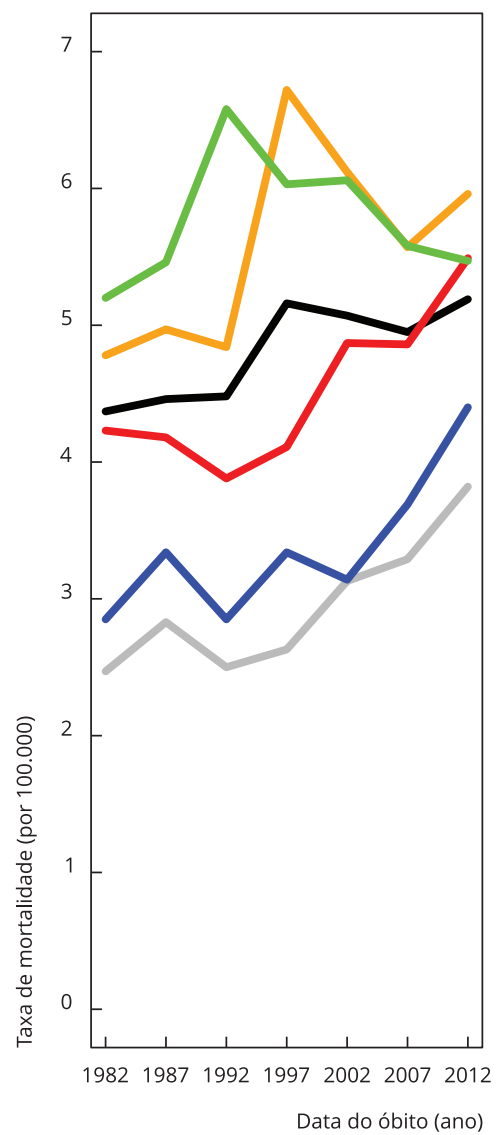

1c) Coorte

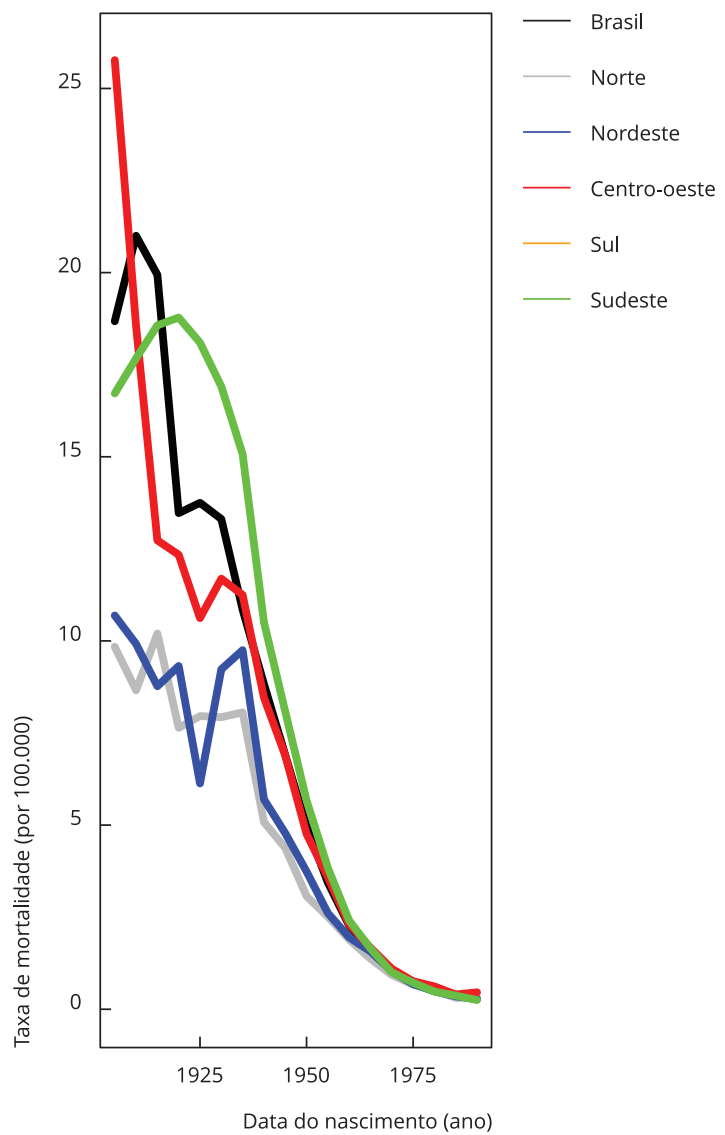


redução progressiva nos demais períodos (Figura 1b). Nas coortes de nascimento verificou-se redução progressiva nas taxas de mortalidade nas coortes de nascimento mais jovens (Figura 1c).

Segundo o modelo BAPC univariado estimado para o Brasil, os riscos de morte por câncer do ovário segundo a idade variaram de 0,09 a 6,10. O risco de morrer por essa patologia dos 20-24 aos 40-44 anos foi menor do que 1, tornando-se positivo e crescente a partir de 45-49 anos (risco relativo > 1) (Figura 2a). Os riscos de óbito por câncer do ovário segundo período variaram de 0,78 a 1,27, no entanto, não observou-se efeito de período significativo em nenhum dos sete quinquênios analisados (Figura 2b). O efeito segundo as coorte de nascimento apontou riscos de óbito variando de 0,60 a 1,49. O risco de óbito foi menor que 1 (risco relativo < 1) para as pessoas nascidas entre 1905 e 1924, e não significativo para mulheres nascidas na coorte de 1925-1929. No entanto, houve tendência crescente do risco de morte, sendo maior que 1 e significativo para as gerações nascidas entre 1930 e 1964 . Nas coortes seguintes verificou-se risco não significativo, porém com tendência de redução para mulheres nascidas entre 1965-1969 e 1990-1994 (Figuras 2c). Os riscos referentes ao efeito aleatório que incorporou a superdispersão dos dados foram significativos e variaram de 0,21 a 1,80.

A Tabela 1 apresenta os resultados do modelo BAPC multivariado considerando as regiões geográficas. O modelo com os efeitos de período e de coorte de nascimento variando segundo as regiões geográficas foi o que melhor se ajustou aos dados (Modelo 7a).

Nas Figuras 3 e 4 observam-se as mudanças no risco de óbito por câncer do ovário entre as regiões brasileiras, segundo períodos e coortes de nascimento, após o ajuste do BAPC multivariado. Verifica-se que o risco de óbito por câncer do ovário varia entre as regiões geográficas brasileiras. No Norte e no Sudeste não observou-se efeito de período significativo, diferentemente do Centro-oeste e Nordeste, em que o risco foi positivo no último quinquênio estudado (2010-2014), e da Região Sul que apresentou aumento do risco de morte a partir do período de 1995-1999 (Figura 3).

Segundo o efeito de coorte de nascimento, o risco de morte foi negativo e significativo na Região Centro-oeste para mulheres nascidas entre 1910 e 1925. Não houve efeito para as demais coortes. Nas regiões Nordeste e Norte, o risco de óbito foi negativo e significativo para mulheres nascidas em coortes mais antigas, mantendo este perfil até a coorte de 1925-1929 no Nordeste, e até 1935-1939 na Região Norte. Inversamente, nas regiões Sudeste e Sul o risco de óbito por esse câncer foi negativo em mulheres nascidas na geração de 1965-1969 em diante (Figura 4). Verificou-se, ainda, aumento progressivo e significativo do risco de morte por câncer do ovário nas regiões Norte e Nordeste, para pessoas nascidas entre 1955 e 1984, e entre 1945 e 1979, respectivamente. Já nas regiões Sul e Sudeste, o risco de morrer por câncer do ovário foi maior entre as gerações mais velhas, sendo nesta ordem, 1915 a 1944 e entre 1920 a 1954 (Figura 4).

\section{Discussão}

Os principais achados deste estudo mostram diferença consistente na magnitude das taxas entre as grandes regiões brasileiras, com aumento progressivo da mortalidade com o avançar da idade em todas as regiões. Há um maior risco de morte para o quinquênio mais recente nas regiões Centro-oeste e Nordeste, para as gerações mais jovens nas regiões Norte e Nordeste, e para as gerações mais antigas no Sul e Sudeste. Esses resultados podem estar relacionados à polarização regional que marca a transição epidemiológica brasileira, que por sua vez tem relação, além da diferença na velocidade com que a dinâmica demográfica se estabelece nas regiões, com disparidades importantes no acesso aos serviços de atenção oncológica 5,15,24,25,26.

Sobre esse aspecto, convém destacar que nas regiões Sudeste, Sul e Centro-oeste a transição demográfica encontra-se mais adiantada em comparação às regiões Norte e Nordeste, e assim o centro-sul brasileiro apresenta estrutura etária mais envelhecida, aumentando o risco de adoecimento e morte por doenças crônicas não transmissíveis 25 . Além disso, discute-se que as localidades com IDH baixo estão mais expostas aos cânceres associados à infecção (câncer do colo do útero, fígado, linfoma não-Hodgkin, entre outros), e as regiões com mais alto IDH aos cânceres associados aos fatores de risco relacionados aos hábitos e estilo de vida, devido às disparidades no processo de urbanização, industrialização e acesso aos serviços de saúde observados entre estas localidades 6,24. Corroborando esses achados, um estudo realizado no Brasil, ao demonstrar o processo de transição do câncer no 


\section{Figura 2}

Risco de mortalidade estimado para câncer do ovário e intervalos de credibilidade (95\%) (IC95\%) para o efeito da idade, período e coorte de nascimento. Brasil, 1980 a 2014.
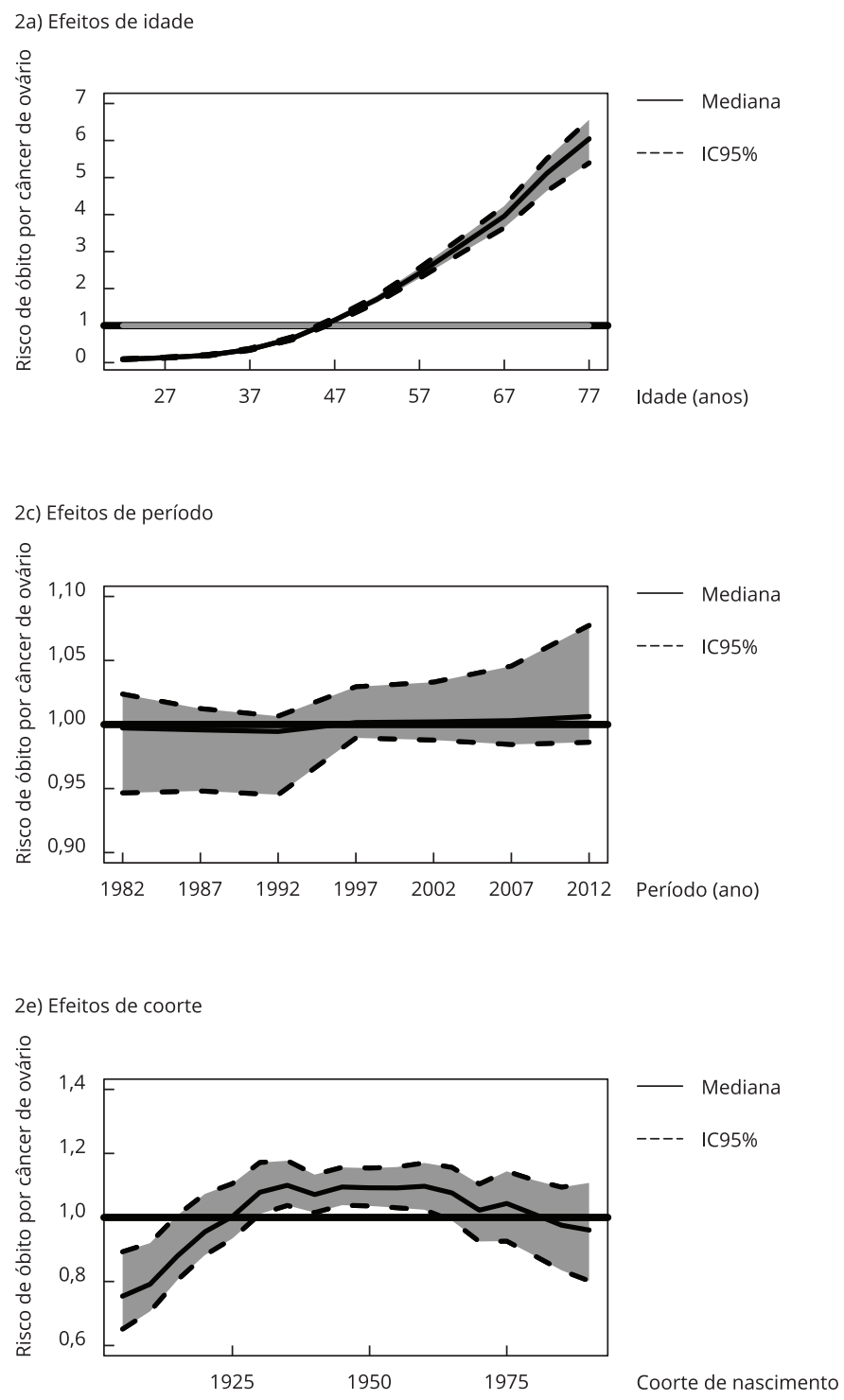

2b) Efeitos de idade

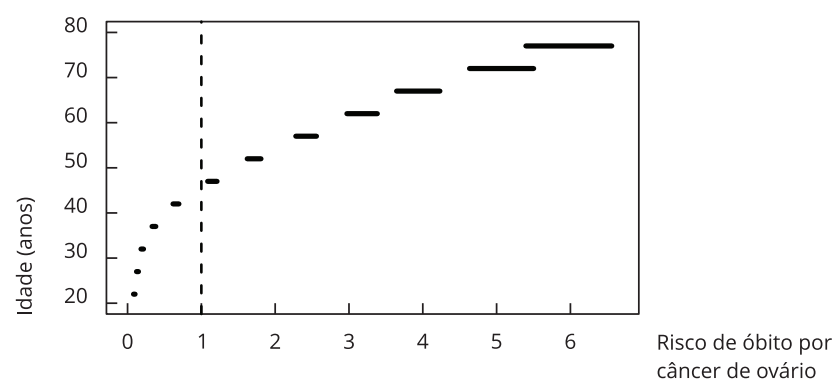

2d) Efeitos de período

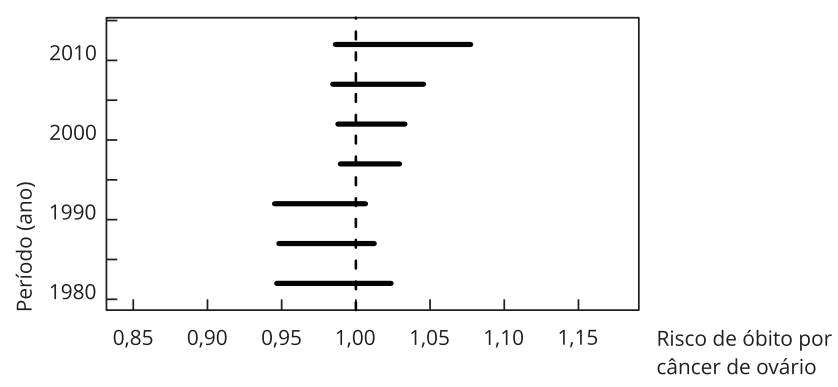

2f) Efeitos de coorte

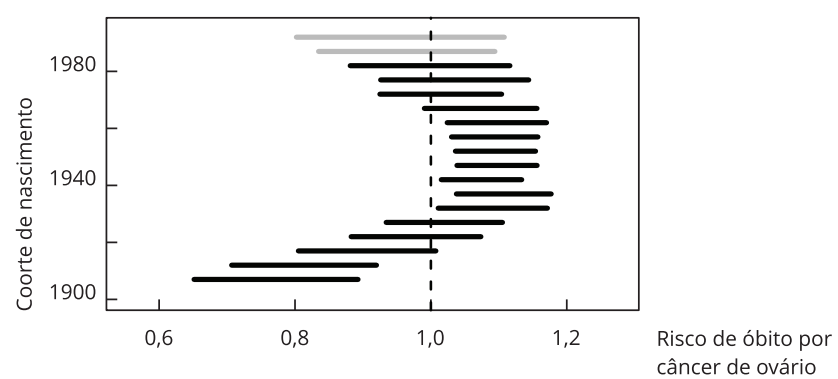

país, utilizou como exemplos os casos do câncer de mama e de colo do útero, e apontou que maiores taxas por câncer de mama são observadas nas localidades com maior desenvolvimento socioeconômico, e nas regiões menos desenvolvidas maiores taxas de mortalidade por câncer do colo do útero são registradas 27.

Outras questões a ser consideradas na avaliação da evolução da mortalidade são a cobertura e a qualidade dos sistemas de informações registradas no país. Ainda que a completude seja superior a $70 \%$ em todas as regiões do Brasil, ela varia entre $72 \%$ no Nordeste e $97 \%$ na Região Sul 28 . Isso significa dizer que, ainda que sejam consideradas razoáveis, com aumento da confiança na utilização das estatísticas, é preciso minimizar o efeito da falta de validade de conteúdo das causas registradas de morte. Razão pela qual a correção de óbitos foi realizada. Nos registros de base populacional brasileiros, a concordância entre a informação das declarações de óbito e dos prontuários, para câncer de 


\section{Tabela 1}

Comparação dos modelos multivariados com os efeitos temporais, efeito aleatório para superdispersão e heterogeneidade por região geográfica (prioris RW1 para os efeitos temporais).

\begin{tabular}{lccccccc}
\hline & Modelo 1 & Modelo 2 & Modelo 3 & Modelo 4 & Modelo 5 & Modelo 6 & Modelo 7 * \\
\hline Log-score & 4,35 & 4,28 & 4,32 & 4,22 & 4,23 & 4,28 & 4,20 \\
DIC & 713,39 & 696,66 & 713,5 & 693,3 & 694,6 & 701,24 & 691,98 \\
\hline
\end{tabular}

DIC: Critério de Informação Deviance.

* Modelo escolhido.

\section{Figura 3}

Estimativas de risco de morte por câncer do ovário, segundo o efeito de período e intervalo de confiança, entre as regiões geográficas brasileiras, no período de 1980 a 2014, após o ajuste do modelo BAPC multivariado.

3a) Efeitos de período - Norte

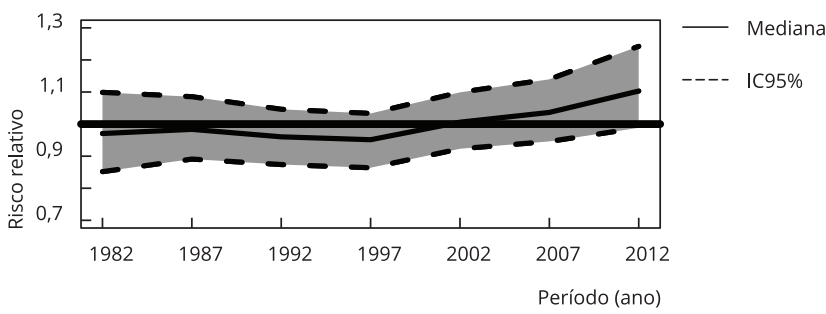

3c) Efeitos de período - Centro-oeste

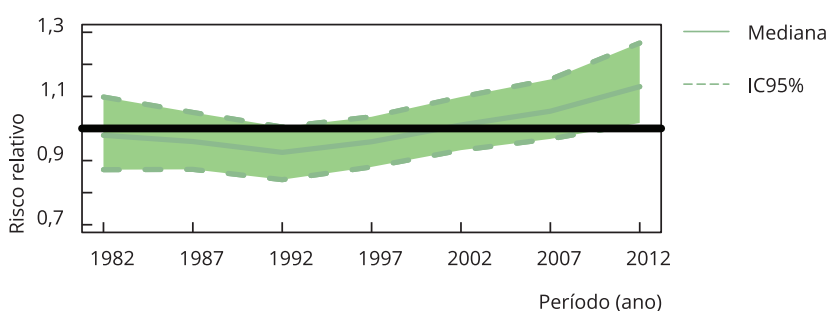

3e) Efeitos de período - Sudeste

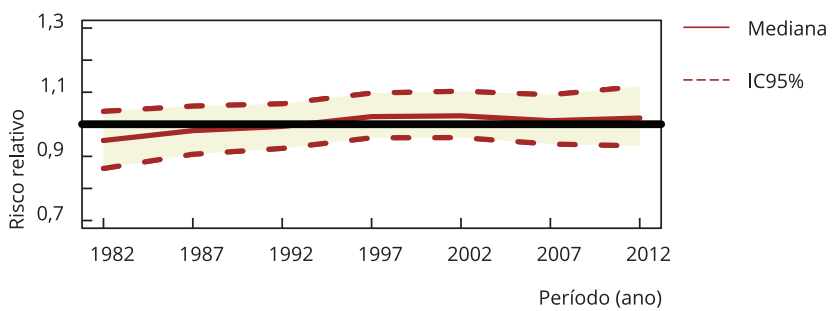

3b) Efeitos de período - Nordeste

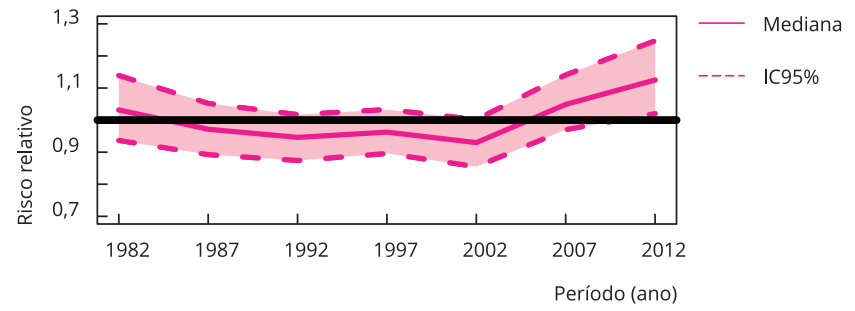

3d) Efeitos de período - Sul

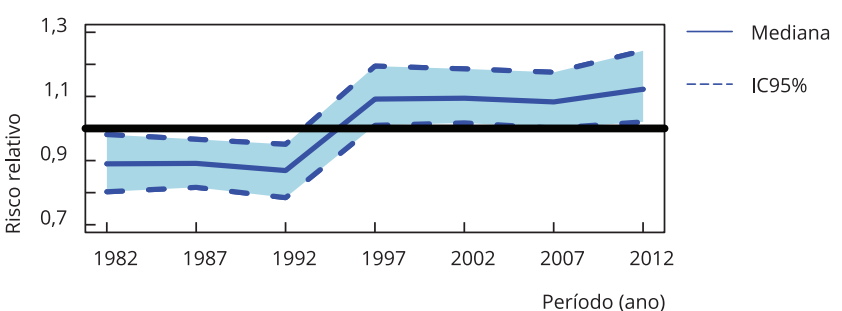

3f) Mediana a posteriori - efeitos de período

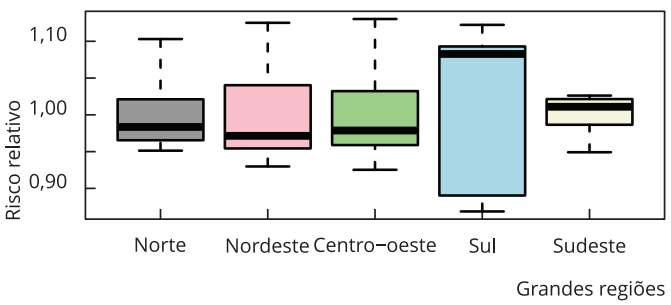




\section{Figura 4}

Estimativas de risco de morte por câncer do ovário, segundo o efeito de coorte e intervalo de confiança, entre as regiões geográficas brasileiras, no período de 1980 a 2014, após o ajuste do modelo BAPC multivariado.

4a) Efeitos de coorte - Norte

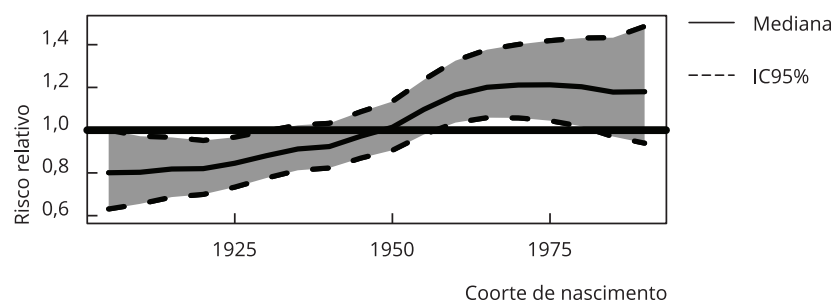

4c) Efeitos de coorte - Centro-oeste

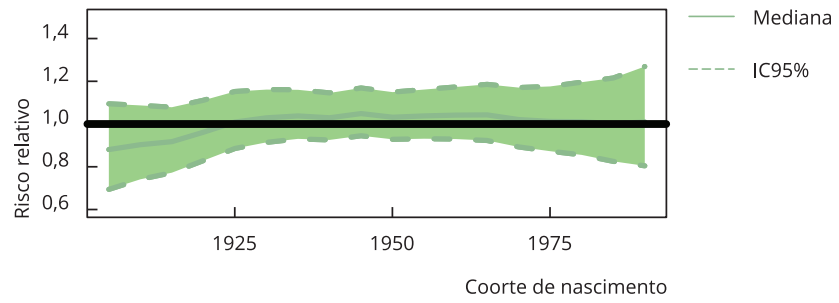

4e) Efeitos de coorte - Sudeste

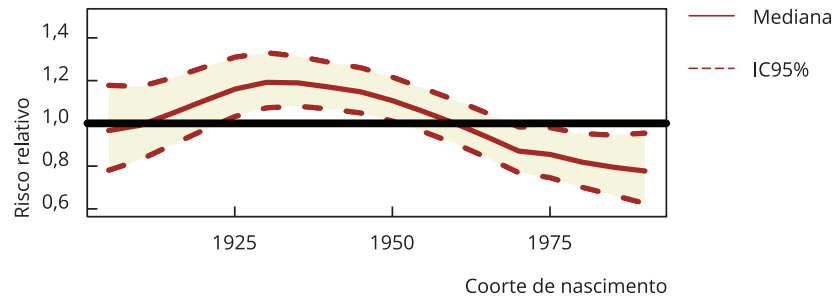

4b) Efeitos de coorte - Nordeste

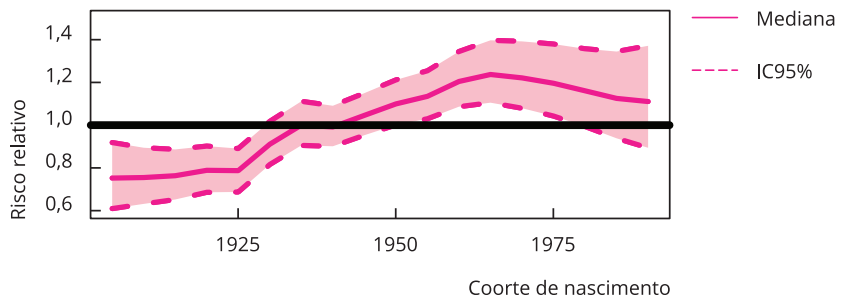

4d) Efeitos de coorte - Sul

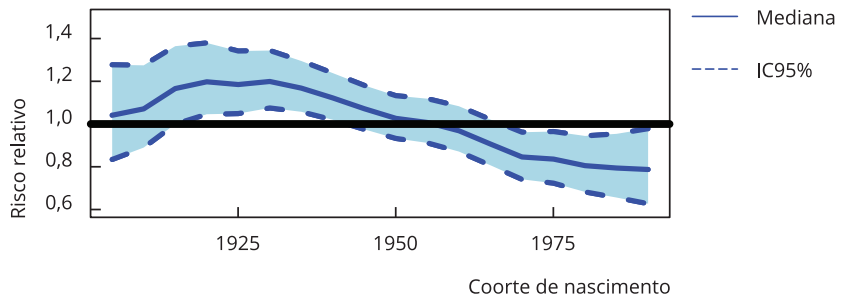

4f) Mediana a posteriori - efeitos de período

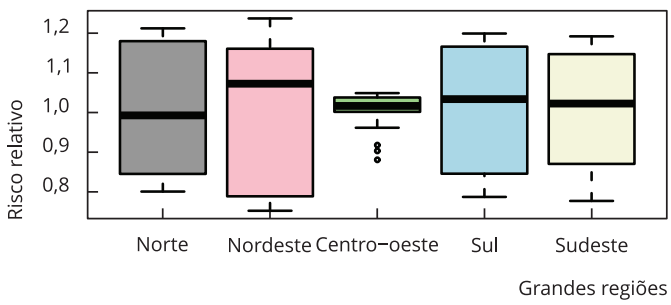

IC95\%: intervalo de credibilidade (95\%); BAPC: modelo período-coorte de nascimento.

ovário, é em torno de $96 \%$ 29. Dessa forma, ainda que haja a preocupação em corrigir os óbitos para nivelar as regiões, o esforço principal dessa técnica é corrigir a magnitude dos óbitos 30 .

No presente estudo, houve aumento da mortalidade com o avançar da idade, resultado esperado por tratar-se de uma doença crônica que se manifesta após a exposição de longa duração a fatores de risco ${ }^{5}$. As mulheres idosas podem apresentar condições clínicas desfavoráveis, aumentando o risco de complicações durante o tratamento oncológico, reduzindo a taxa de sobrevida 4,5,7,9,12,13,14,31,32. Além disso, grande proporção delas são diagnosticadas em estágios avançados 33,34,35.

No que diz respeito ao efeito de período, no Brasil e nas regiões Norte e Sudeste não houve efeito significativo. Nas regiões Nordeste e Centro-oeste houve aumento do risco no último período da série histórica, e na Região Sul observou-se elevação do risco a partir de 1995-1999. Acredita-se que o efeito de período observado nas regiões Centro-oeste, Nordeste e Sul deva-se à diferença 
na efetividade da implantação de políticas públicas, incluindo as formas de organização da regionalização para a ampliação do acesso aos serviços de saúde, e ações mais pontuais para a saúde da mulher, no que se refere à disponibilização de métodos contraceptivos hormonais, e orientações de planejamento familiar 25,26 .

Os resultados deste estudo diferem dos observados em países desenvolvidos que vivenciaram redução nas taxas de mortalidade por câncer do ovário, especialmente a partir da década de 1990 $7,8,9,13,14,31,32$, devido às melhorias no diagnóstico da doença, ampliação do acesso aos exames de imagem, combinações mais efetivas de quimioterápicos (platinas com taxanos), além de cirurgias mais agressivas (debulking), que diminuíram a taxa de reicidiva da doença 7,8,9,13,14,31,32.

De fato, a elevação do risco de morte por câncer do ovário na Região Sul a partir de meados da década de 1990 e no último período da série histórica nas regiões Norte e Centro-oeste, talvez possa estar correlacionada à expansão da atenção à saúde, observada no Brasil após a implantação do Sistema Único de Saúde (SUS), ampliando o acesso aos exames de imagem como ultrassonografia de abdômen e transvaginal, que possivelmente aumentaram a possibilidade do diagnóstico da patologia e melhoraram a certificação dos registros de óbito. Contudo, a ampliação do acesso aos serviços de saúde não foi acompanhada de redução nas taxas de mortalidade devido à característica dessa doença, que se manifesta de maneira insidiosa 36,37. Desse modo, a maioria das mulheres é diagnosticada em estágios avançados, contribuindo para a baixa taxa de sobrevida observada no Brasil no período de 2005 a 2009 (31,8\%; IC95\%: 29,5-40,3) 34; 2010 a 2014 (34,9\%; IC95\%: 29,5-40,3) 35.

O efeito de coorte de nascimento no Brasil e nas regiões Nordeste e Norte assemelha-se ao observado na Grécia e Espanha, onde as coortes de nascimento mais antigas apresentam menor risco de morte por câncer do ovário, com o aumento do risco para as gerações mais jovens ${ }^{37}$, já os resultados das regiões Sul e Sudeste são semelhantes aos verificados nos países nórdicos, Reino Unido, Japão e Canadá, onde o risco de morrer por câncer do ovário foi maior nas coortes mais antigas, com redução progressiva importante para mulheres de coortes mais jovens 12,13,37,38.

Os autores afirmam que o maior risco de morte por câncer do ovário observado em mulheres nascidas entre a virada do século XX até a década de 1920 esteve correlacionado à queda da taxa de fecundidade e natalidade ocorrida no início daquele século, devido às duas grandes guerras mundiais 12,13,37,38. Em sentido contrário, as menores taxas de mortalidade e risco de morte por câncer do ovário, para mulheres nascidas após a década de 1920, são atribuídos ao aumento da taxa de fecundidade e natalidade, o chamado baby boom ocorrido após as duas grandes guerras 12,13,37,38. E também, advogam que o aumento da taxa de fecundidade, associado à introdução e ampliação da utilização de contraceptivos orais, sobretudo, em mulheres nascidas a partir de 1940, possam ter contribuído para a redução da incidência e mortalidade por câncer do ovário nas gerações após a década de 1930 12,13,37,38.

A relação entre os fatores reprodutivos e o câncer do ovário foi comprovada em estudos observacionais em nível individual, e estas associações permanecem em revisões sistemáticas com metanálise 39,40. As alterações genéticas na superfície epitelial do ovário ocorrem como resposta a uma incessante ovulação, por microtraumas ou injúrias de repetição, e quanto maior for o período de permanência em anovulação em uma mulher, maior será a proteção do epitélio ovariano a processos carcinogênicos. Os períodos de anovulação correspondem à gravidez, à amamentação, ao uso de anticoncepcionais orais e ao pequeno período menstrual (menarca tardia e menopausa precoce) 39,40 . Outra fator de proteção para esse câncer é a ligadura de trompas. Acredita-se que o procedimento cirúrgico impeça a entrada de corpos estranhos provenientes do canal vaginal nos ovários, e assim impediria o processo de carcinogênese 41 .

O menor risco de morte observado nas coortes de nascimento mais velhas no Brasil e regiões Norte, Nordeste e Centro-oeste, assim como ocorreu com as gerações após a década de 1920 no Reino Unido 12, pode ser explicado em parte pela alta taxa de natalidade e fecundidade observada nestas gerações, já o posterior aumento do risco estimado de óbito por câncer do ovário nas coortes mais jovens pode correlacionar-se à redução nas taxas de fecundidade nas sucessivas gerações 42, que não foi acompanhado pelo aumento da prevalência de consumo de anticoncepcional oral como método contraceptivo, suficiente para compensar o risco de adoecimento gerado pela diminuição de período de anovulação, promovido pelas gestações 42,43.

Adicionalmente a essa realidade, as coortes mais jovens estiveram mais expostas a importantes fatores de risco para o câncer do ovário, tais como mudanças nos hábitos alimentares, redução da 
prática de atividade física e aumento da prevalência do tabagismo 30,44. Outra questão que merece destaque foi a redução do período de amamentação vivenciada por mulheres das coortes mais jovens, tanto pela redução da fecundidade quanto pela introdução e aumento progressivo da oferta de fórmulas lácteas e leites em pó artificiais pela indústria alimentícia, e a redução da prevalência de amamentação a partir dos anos 1930 e 1940 30,44.

O padrão diferenciado do risco de morte nas regiões Sul e Sudeste em relação às outras regiões tem como uma das possíveis explicações o melhor acesso aos serviços de saúde nestas localidades 26. Espera-se que as mulheres residentes nessas regiões, em todas as coortes de nascimento, tenham maior possibilidade de diagnóstico correto, gerando registros de óbito de melhor qualidade, haja vista a menor proporção de óbitos classificados como câncer geral de diagnóstico incompleto, em comparação às demais regiões geográficas 18. As mulheres dessas regiões, mesmo nas coortes mais velhas, teriam maior risco de morrer por câncer do ovário devido ao efeito do período de acesso aos serviços de saúde, tendo em vista que a maioria dos serviços especializados em atenção oncológica encontra-se nas regiões Sul e Sudeste do Brasil 26. As diferenças no risco entre as gerações mais velhas e jovens nessas regiões também podem ser explicadas pelo fato de as mulheres mais idosas terem maior probabilidade de serem diagnosticadas em estágios avançados, reduzindo a sua sobrevida global e sobrevida livre de doença.

Acredita-se que o menor risco de morte nas coortes de nascimento mais jovens no Sul e Sudeste correlaciona-se à introdução e ampliação do uso do anticoncepcional oral e aumento da prevalência de mulheres em idade fértil realizando cirugia de ligadura de trompas 12,13,14,33,37,42. Contribuindo para a redução da incidência e mortalidade por esse câncer, em que pese a elevação da prevalência na população feminina de outros fatores de risco associados ao câncer do ovário, tais como hábitos alimentares não saudáveis, sedentarismo, redução na prevalência de amamentação, e aumento na prevalência de tabagismo 30,44,45.

Finalmente, os resultados deste estudo devem ser analisados com cautela, as taxas de mortalidade sofrem influência da qualidade da informação dos registros de mortalidade. Buscou-se obter taxas de mortalidade mais fidedignas por meio das correções dos óbitos, no entanto, não foram corrigidos os problemas associados à cobertura do SIM. Outra limitação refere-se aos registros de óbito não apresentarem especificação do tipo histológico, que seria de grande importância na estratificação da avaliação da mortalidade, uma vez que há diferenças nas faixas etárias acometidas de acordo com o tipo histológico, e pouco se sabe sobre a etiologia dos tipos histológicos não epiteliais (células germinativas e cordão sexual estromal).

Este é o primeiro estudo brasileiro que avaliou a evolução da mortalidade por câncer do ovário segundo os efeitos temporais simultâneos de idade, período e coorte, com base em modelos probabilísticos, identificando o risco estimado de morte segundo cada efeito e sua heterogeneidade entre as regiões geográficas do país. Contribuindo, assim, para o Plano de Ações Estratégicas para o Enfrentamento das Doenças Crônicas Não Transmissíveis no Brasil, especificamente no componente de vigilância da mortalidade, fornecendo evidências para o planejamento e avaliação de políticas de saúde para a referida doença nas distintas regiões do país.

\section{Conclusão}

A avaliação da evolução da mortalidade por câncer do ovário segundo os efeitos temporais simultâneos de idade, período e coorte de nascimento, com base em modelos probabilísticos, identificou heterogeneidade no risco estimado de morte segundo período e coorte nas distintas regiões brasileiras. A análise do efeito APC revelou aumento do risco de morte por câncer do ovário entre mulheres brasileiras, em todas as regiões geográficas, a partir da quarta década de vida. Houve aumento do risco de morte para as regiões Centro-oeste, Norte e Sul no período de 2010 a 2014. Além disso, foi verificado efeito de coorte de nascimento, com aumento do risco médio de morte para o Brasil como um todo, nas regiões Norte e Nordeste, entre coortes de nascimento mais jovens e nas regiões Sul e Sudeste nas coortes mais antigas.

É possível supor que esses resultados, considerando-se o fato de que são fatores semelhantes para incidência e mortalidade, são associados aos distintos processos de mudanças do comporta- 
mento reprodutivo das mulheres residentes nessas localidades, especialmente no que diz respeito à paridade e ao uso de anticoncepcional oral, bem como devido às diferentes fases do processo de transição demográfica e às disparidades de acesso aos serviços de atenção oncológica observados no território nacional.

\section{Colaboradores}

K. C. Meira, J. Santos e T. C. Simões contribuíram com a concepção e projeto, análise e interpretação dos dados, redação, revisão crítica relevante do conteúdo intelectual e com a aprovação final da versão a ser publicada. C. M. F. P. Silva contribuiu com a concepção e projeto, análise e interpretação dos dados e com a aprovação final da versão a ser publicada. A. A. Ferreira contribuiu com a concepção e projeto, análise e interpretação dos dados, redação e revisão crítica relevante do conteúdo intelectual. R. M. Guimarães contribuiu com a redação, revisão crítica relevante do conteúdo intelectual e com a aprovação final da versão a ser publicada.

\section{Informações adicionais}

ORCID: Karina Cardoso Meira (0000-0002-17225703); Juliano dos Santos (0000-0001-9961-3576); Cosme Marcelo Furtado Passos da Silva (00000001-7789-1671); Aline Alves Ferreira (00000001-5081-3462); Raphael Mendonça Guimarães (0000-0003-1225-6719); Taynãna César Simões (0000-0002-5849-343X).

\section{Agradecimentos}

Ao Conselho Nacional de Desenvolvimento Científico e Tecnológico (CNPq) e à Coordenação de Aperfeiçoamento de Pessoal de Nível Superior (Capes; código de financiamento 001).

\section{Referências}

1. Ferlay J, Soerjomataram I, Dikshit R, Eser S, Mathers C, Rebelo M, et al. Cancer incidence and mortality worldwide: sources, methods and major patterns in GLOBOCAN 2012. Int J Cancer 2015; 136:E359-86.

2. Azevedo e Silva G, de Moura L, Curado MP, Gomes FS, Otero U, Rezende LFM, et al. The fraction of cancer attributable to ways of life, infections, occupation, and environmental agents in Brazil in 2020. PLoS One 2016; 11:e0148761.

3. Sopik V, Iqbal J, Rosen B, Narod SA. Why have ovarian cancer mortality rates declined? Part I. Incidence. Gynecol Oncol 2015; 138:741-9.

4. Sopik V, Iqbal J, Rosen B, Narod SA. Why have ovarian cancer mortality rates declined? Part II. Case-fatality. Gynecol Oncol 2015; 138:750-6.

5. Sopik V, Rosen B, Giannakeas V, Narod SA. Why have ovarian cancer mortality rates declined? Part III. Prospects for the future. Gynecol Oncol 2015; 138:757-61.

6. Bray F, Jemal A, Grey N, Ferlay J, Forman D. Global cancer transitions according to the Human Development Index (2008-2030): a population-based study. Lancet Oncol 2012; 13:790-801.

7. Instituto Nacional de Câncer José Alencar Gomes da Silva. Estimativa da incidência do câncer no Brasil, 2018. Rio de Janeiro: Instituto Nacional de Câncer José Alencar Gomes da Silva; 2018.

8. Barbosa IR. Tendências e projeções da mortalidade pelos cânceres específicos ao gênero no Brasil [Tese de Doutorado]. Natal: Universidade Federal do Rio Grande do Norte; 2015. 
9. Chiaffarino F, Parazzini F, Bosetti C, Franceschi S, Talamini R, Canzonieri V, et al. Risk factors for ovarian cancer histotypes. Eur J Cancer 2007; 43:1208-13.

10. Reid BM, Permuth JB, Sellers TA. Epidemiology of ovarian cancer: a review. Cancer Biol Med 2017; 14:9-32.

11. Park S, Shin HR, Shin A, Yeo Y, Choi JY, Jung $\mathrm{KW}$, et al. Population attributable risks of modifiable reproductive factors for breasts and ovarian cancers in Korea. BMC Cancer 2016; 16:5.

12. dos Santos Silva I, Swerdlow AJ. Recent trends in incidence of and mortality from breast, ovarian and endometrial cancers in England and Wales and their relation to changing fertility and oral contraceptive use. Br J Cancer 1995; 72:485-92.

13. Zhang J, Ugnat AM, Clarke K, Mao Y. Ovarian cancer histology-specific incidence trends in Canada 1969-1993: age-perid-cohort analyses. Br J Cancer 1999; 81:152-8.

14. Wang B, Liu SZ, Zheng RS, Zhang F, Chen WQ, Sun XB. Time trends of ovarian cancer in China. Asian Pac J Cancer Prev 2014; 15:191-3.

15. Holford TR. Undestranding the effects of age, period, and cohort on incidence and mortality rates. Annu Rev Public Health 1991; 12:425-57.

16. Riebler A, Held L, Rue H. Estimation and extrapolation of time trends in registry data borrowing strength from related populations. Ann Appl Stat 2012; 6:304-33.

17. Mathers CD, Bernard C, Iburg KM, Inoue M, Fat DM, Shibuya K, et al. Global Burden of Disease in 2002: data sources, methods and results. Geneva: World Health Organization; 2003. (Global Programme on Evidence for Health Policy Discussion Paper, 54).

18. Giusti ACB, Salvador PTCO, Santos J, Meira KC, Camacho AR, Guimarães RM, et al. Trends and predictions for gastric cancer mortality in Brazil. World J Gastroenterol 2016; 22:652738.

19. Boyle P, Parkin DM. Statistical methods for registries. In: Jensen OM, Parkin DM, MacLennan R, Muir CS, Skeet RG, editors. Cancer registration principles and methods. Lyon: International Agency for Research on Cancer; 1991.p. 126-58.

20. Knorr-Held L, Rainer E. Projections of lung cancer mortality in West Germany: a case study in Bayesian prediction. Biostatistics 2001; 2:109-29.

21. Riebler A, Held L, Rue H. Correlated GMRF priors for multivariate age-period-cohort models. In: Bowman A, editor. Proceedings of the 25th International Workshop on Statistical Modelling. Glasgow: University of Glasgow; 2010. p. 1-6.

22. Spiegelhalter DJ, Best NG, Carlin BP, van der Linde A. Bayesian measures of model complexity and fit. J R Stat Soc Series B Stat Methodol 2002; 64:583-616.

23. Gneiting T, Raftery AE. Strictly proper scoring rules, prediction, and estimation. J Am Stat Assoc 2007 ; 102:359-78.
24. Bray F, Soerjomataram I. The changing global burden of cancer: transitions in human development and implications for cancer prevention and control. In: Gelband H, Jha P, Sankaranarayanan R, Horton S, editors. Cancer: disease control priorities. v. 3. 3rd Ed. Washington DC: The International Bank for Reconstruction and Development/The World Bank; 2015. p. 5-25.

25. Vasconcelos AMN, Gomes MMF. Transição demográfica: a experiência brasileira. Epidemiol Serv Saúde 2012; 21:539-48.

26. Oliveira EXG, Melo ECP, Pinheiro RS, Noronha $\mathrm{CP}$, Carvalho MS. Acesso à assistência oncológica: mapeamento dos fluxos origemdestino das internações e dos atendimentos ambulatoriais. O caso do câncer de mama. Cad Saúde Pública 2011; 27:317-26.

27. Guimarães RM, Muzi CD, Teixeira MP. A transição da mortalidade por cânceres no Brasil e a tomada de decisão estratégica nas políticas públicas de saúde da mulher. Revista de Políticas Públicas 2016; 20:33-50.

28. França E, de Abreu DX, Rao C, Lopez AD. Evaluation of cause-of-death statistics for Brazil, 2002-2004. Int J Epidemiol 2008; 37:891901.

29. Oliveira PPV, Azevedo e Silva G, Curado MP, Malta DC, Moura L. Confiabilidade da causa básica de óbito por câncer entre Sistema de Informações sobre Mortalidade do Brasil e Registro de Câncer de Base Populacional de Goiânia, Goiás, Brasil. Cad Saúde Pública 2014; 30:296-304.

30. Departamento de Vigilância de Doenças e Agravos Não Transmissíveis e Promoção da Saúde, Secretaria de Vigilância em Saúde, Ministério da Saúde. Saúde Brasil 2014: uma análise da situação de saúde e das causas externas. Brasília: Ministério da Saúde; 2015.

31. Howlader N, Noone AM, Krapcho M, Miller D, Bishop K, Altekruse SF, et al. SEER Cancer Statistics Review, 1975-2013. Bethesda: National Cancer Institute. http://seer.cancer.gov/ csr/1975_2013/(acessado em 19/Mai/2018).

32. Maas HA, Kruitwagen RF, Lemmens VE, Goey $\mathrm{SH}$, Janssen-Heijnen ML. The influence of age and co-morbidity on treatment and prognosis of ovarian cancer: a population-based study. Gynecol Oncol 2005; 97:104-9.

33. Tamakoshi K, Kondo T, Yatsuya H, Hori Y, Kikkawa F, Toyoshima H. Trends in the mortality (1950-1997) and incidence (1975-1993) of malignant ovarian neoplasm among Japanese women: analyses by age, time, and birth cohort. Gynecol Oncol 2001; 83:64-71.

34. Allemani C, Matsuda T, Di Carlo V, Harewood R, Matz M, Nikšić M, et al. Global surveillance of trends in cancer survival 2000-14 (CONCORD-3): analysis of individual records for 37513025 patients diagnosed with one of 18 cancers from 322 population-based registries in 71 countries. Lancet 2018; 391:102375 . 
35. Allemani C, Weir HK, Carreira H, Harewood R, Spika D, Wang XS, et al. Global surveillance of cancer survival 1995-2009: analysis of individual records for $25,676,887$ patients from 279 population-based registries in 67 countries (CONCORD-2). Lancet 2015; 385:9771010.

36. Moss HA, Berchuck A, Neely ML, Myers ER, Havrilesky LJ. The United States: secondary analysis of the UK Collaborative Trial of Ovarian Cancer Screening (UKCTOCS). JAMA Oncol 2018; 4:190-5.

37. González-Diego P, López-Abente G, Pollán M, Ruiz M. Time trends in ovarian cancer mortality in Europe (1955-1993): effect of age, birth cohort and period of death. Eur J Cancer 2000; 36:1816-24.

38. Hirabayashi Y, Marugame T. Comparison of time trends in ovary cancer mortality (19902006) in the world, from the WHO Mortality Database. Jpn J Clin Oncol 2009; 39:860-1.

39. Luan NN, Wu QJ, Gong TT, Vogtmann E, Wang YL, Lin B. Breastfeeding and ovarian cancer risk: a meta-analysis of epidemiologic studies. Am J Clin Nutr 2013; 98:1020-31.

40. Gong TT, Wu QJ, Vogtmann E, Lin B, Wang YL. Age at menarche and risk of ovarian cancer: a meta-analysis studies. Int J Cancer 2013; 132:2894-900
41. Madsen C, Baandrup L, Dehlendorff C, Kjaer SK. Tubal ligation and salpingectomy and the risk of epithelial ovarian cancer and borderline ovarian tumors: a nationwide case-control study. Acta Obstet Gynecol Scand 2015; 94:8694.

42. Ministério da Saúde. Pesquisa Nacional de Demografia e Saúde da Criança e da Mulher PNDS 2006: dimensões do processo reprodutivo e da saúde da criança. Brasília: Ministério da Saúde; 2009. (Série G. Estatística e Informação em Saúde).

43. Martine G. Brazil's fertility decline 1965-95: a fresh look at key factors. Popul Dev Rev 1996; 22:47-75.

44. Kuhlmann Jr. M, Magalhães MGS. A infância nos almanaques: nacionalismo, saúde e educação (Brasil 1920-1940). Educ Rev 2010; 26:327-49.

45. Sena MCF, Silva EF, Pereira MG. Tendência do aleitamento materno no Brasil no último quarto do século XX. Rev Bras Epidemiol 2007; 10:499-505. 


\section{Abstract}

This article sought to evaluate the effects of age, period and birth cohort (APC) on the temporal evolution of mortality due to ovarian cancer in Brazil and its regions from 1980 to 2014. This is an ecological, time-trend study using APC models with a Baysean approach and INLA (Integrated Nested Laplace Approximations) deterministic method for parameter inference. Mortality data and population data were obtained from the Brazilian Health Informatics Department. Rates of mortality due to ovarian cancer, according to geographical region, were standardized using the direct method, after correcting deaths for undefined causes and incomplete cancer diagnosis. In the period we studied, Brazil had 4.91 deaths due to ovarian cancer per 100,000 women, the Southern (5.66) and Southeastern regions (5.70) had higher rates per 100,000 women and the Northern region had the lowest rate (3.13/100,000 women). In all regions, there was a progressive increase in mortality as ages advanced. The multivariate best fit APC model showed positive risk of death in the Central and Northeast between 2010-2014 and, beginning in 1995-1999, in the South. We also observed a positive and significant risk of death for older cohorts in the South and Southeast and a reduced risk for younger cohorts. The inverse was opposed in the Northern and Northeastern regions. The data shows a heterogeneous pattern in the temporal evolution of mortality due to ovarian cancer in the Brazilian geographical regions, which may be related to the distinct demographic and epidemiological transition processes experienced in these regions.

Ovarian Neoplasms; Mortality; Time Series Studies

\section{Resumen}

El objetivo fue evaluar los efectos de la edad, periodo y cohorte de nacimiento (APC) en la evolución temporal de la mortalidad por cáncer de ovario en Brasil y sus macrorregiones, durante el periodo de 1980 a 2014. Es un estudio ecológico de tendencia temporal, donde se utilizaron modelos APC con un enfoque bayesiano y método determinista INLA (Integrated Nested Laplace Approximations) en la inferencia de los parámetros. Los datos de mortalidad y poblacionales se obtuvieron del Departamento de Informática del Sistema Único de Salud. Las tasas de mortalidad por cáncer de ovario, según región geográfica, se estandarizaron mediante el método directo, tras la corrección de los óbitos para causas mal definidas y diagnóstico incompleto de cáncer. Durante el período de estudio, Brasil presentó 4,91 óbitos por cáncer de ovario por cada 100.000 mujeres, las regiones Sur $(5,66)$ y Sudeste $(5,70)$ presentaron las mayores tasas por 100.000 mujeres, y la región Norte la menor tasa (3,13/100.000 mujeres). Hubo un aumento progresivo de la mortalidad con el avance de la edad en todas las regiones. El modelo APC multivariado de mejor ajuste evidenció un riesgo positivo de muerte en el Centro-oeste y Nordeste entre 2010-2014 y, a partir del periodo de 19951999 en la región Sur. Se observó incluso el riesgo positivo y significativo de muerte para las cohortes más antiguas en el Sur y Sudeste, y riesgo reducido para las cohortes más jóvenes. Lo inverso se observó en las regiones Norte y Nordeste. Se evidenció un patrón heterogéneo en la evolución temporal de la mortalidad por cáncer de ovario en las regiones geográficas brasileñas, lo que puede estar relacionado con los distintos procesos de transición demográfica y epidemiológica vividos por estas regiones.

Neoplasias Ováricas; Mortalidad; Estudios de Series Temporales
Recebido em 02/Mai/2018

Versão final reapresentada em 31/Out/2018

Aprovado em 29/Nov/2018 